The conditions under which this degeneracy can be maximized have been explored through specific studies of several different lattice geometries, including a particularly interesting one called the shakti lattice ${ }^{4}$.

The shakti lattice, illustrated in Fig. 1b, is a modification of the square lattice. Using a thermal-annealing protocol, Gilbert et al. ${ }^{1}$ were able to tune the interaction energy to show that the ground state contains three-moment vertices, half of which are relaxed into defect states. The energies of the defects are higher than that of the three-moment vertex ground state. There are two defect vertices per plaquette and these defects can adopt six configurations without changing the energy of the system. In this way, the authors could map ground-state configurations of the shakti lattice exactly onto the six-vertex ice-rule model, and thereby demonstrate that the shakti lattice topology gives rise to an intrinsic - and extensive - degeneracy ${ }^{1}$.

Gilbert et al. ${ }^{1}$ also examined the dynamics of vertices, and searched for evidence of magnetic charge ordering. The three-moment vertices have uncompensated magnetic poles and are charged, suggesting the possibility for long-range interactions between effective charges as studied in other spin-ice systems $^{5}$. Using Monte Carlo simulations of a model Hamiltonian, and accounting carefully for possible effects due to disorder, Gilbert et al. ${ }^{1}$ were able to find strong evidence for interactions between the effective magnetic charge carried by vertices from the experimental data. One especially enticing possibility noted by the authors is that bound states for magnetic charges may exist, suggestive of a magnetic analogue to the polaron.

From its inception, the study of artificial spin ice has claimed an allure by promising to address topics of fundamental scientific interest within entirely new contexts. The physics studied has included topics that range from field theory to nonlinear dynamics: Nambu monopoles ${ }^{6}$, avalanches and dimensional reduction ${ }^{7}$, and returnpoint memory ${ }^{8}$. The work by Gilbert et al. ${ }^{1}$ has created opportunities for studies of fundamental thermodynamics. Most importantly, it represents a first step along a path into an uncharted territory - a landscape of advanced functional materials in which topological effects on physical properties can be explored and harnessed. This is a wonderful place indeed to go a-wandering.

Robert L. Stamps is in the School of Physics and Astronomy at the University of Glasgow, Glasgow G12 8QQ, UK.

e-mail: robert.stamps@glasgow.ac.uk

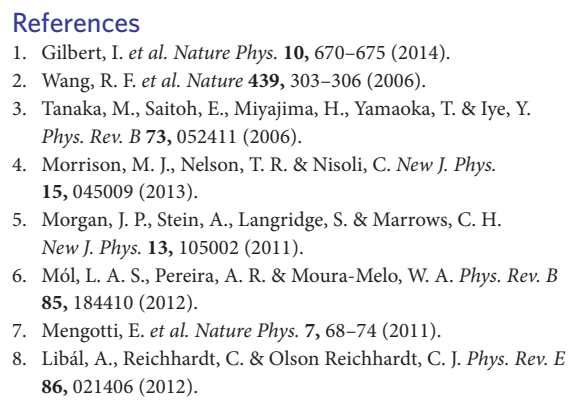

Published online: 3 August 2014

\title{
SPECTROSCOPY
}

\section{Vibrant colours}

Shine light on a molecule and it will generally scatter elastically. But a few photons will bounce off with a different wavelength, having transferred some of their energy to the molecule. This is known as Raman scattering and it involves the excitation of vibrational or rotational transitions - a very handy feature for vibrational spectroscopy, as the wavelength shifts act as molecular fingerprints, allowing for the identification of molecules in a sample. Now, two studies in Nature Photonics have shown that a related technique has potential to improve imaging capabilities - from the single-molecule level all the way up to intact tissues.

Raman scattering and its close cousin, stimulated Raman scattering, enable another spectroscopic technique known as coherent anti-Stokes Raman scattering (CARS), which uses photons of different colours and yields a coherent signal. CARS has been used and studied extensively over the past forty years, but it seems that there is still room for innovation. For instance, CARS is useful for imaging biological tissues, but it does have its limitations - particularly

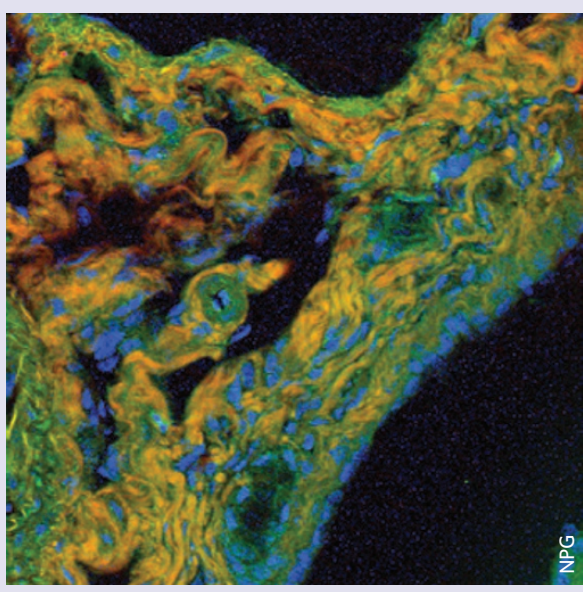

in terms of speed. It is also hampered by distortions owing to the non-resonant background that arises due to electronic excitations that accompany the resonant vibrational modes.

Charles H. Camp Jr and colleagues (Nature Photon. 8, 627-634; 2014) made use of this non-resonant background to amplify weak Raman signals and, by combining this with three-colour excitation, they succeeded in producing high-speed chemical images of liver tissues (pictured). Their technique is expected to find application in fundamental research and clinical studies - and may even lead to further developments in in vivo imaging.

At the other end of the spectrum, probing single molecules in ambient conditions presents a different set of challenges and requires another approach. Amplification of the weak Raman signal from a single molecule can be achieved with surface-enhanced Raman scattering using plasmonic antennas, as Steven Yampolsky and co-workers have shown (Nature Photon. 8, 650-656; 2014). The team attached ethylene molecules to two gold nanospheres in a dumbbell-like structure and enclosed it in silica. With their dumbbell antenna, they were able to use the CARS signal to record the motion of the vibrational wave packet of single molecules in real time. Reaching such sensitivity at room temperature is quite remarkable. CARS may be old, but it is certainly capable of learning some new tricks.

\section{IULIA GEORGESCU}

